\title{
Effect of Michell Function on Steady State Behavior of Thick Circular Plate with Internal Heat Generation
}

\author{
C. M. Bhongade ${ }^{1}$ and M. H. Durge ${ }^{2}$ \\ ${ }^{I}$ Department of Mathematics, Shri. Shivaji College, Rajura, Maharashtra, India \\ ${ }^{2}$ Department of Mathematics, Anand Niketan College, Warora, Maharashtra, India
}

\begin{abstract}
The present paper deals with the determination of displacement and thermal stresses in a thick $(M \neq$ $O$ circular plate under steady temperature field. Arbitrary heat flux is applied at the upper surface of a thick $(M \neq 0)$ circular plate, whereas lower surface at zero temperature and the fixed circular edge is thermally insulated. Here we compute the effects of Michell function on the thickness of circular plate with internal heat generation. The governing heat conduction equation has been solved by the method of integral transform technique. The results are obtained in a series form in terms of Bessel's functions. The results for temperature change, displacement and stresses have been computed numerically and illustrated graphically.
\end{abstract}

Keywords: Thick plate $(M \neq 0)$, thin plate $(M=0)$, thermal stresses, steady state.

\section{Introduction}

The steady state thermal stresses in circular disk subjected to an axisymmetric temperature distribution on the upper face with zero temperature on the lower face and the circular edge has been considered by Nowacki (1957). Roy Choudhary (1972) has determined the quasi-static thermal stresses in thin circular plate. Sharma et al. (2004) studied the behavior of thermoelastic thick plate under lateral loads. Gogulwar and Deshmukh (2005) determined the thermal stresses in thin circular plate with heat sources. Kulkarni and Deshmukh (2008) determined quasi-static thermal stresses in steady state thick circular plate. Deshmukh et al. (2009) studied non homogeneous steady state heat conduction problem in a thin circular plate and discussed its thermal stresses due to its internal heat generation at a constant rate. Recently Deshmukh et al. (2011) has determined the quasi-static thermal stresses due to an instantaneous point heat source in a circular plate subjected to time dependent heat flux at the fixed circular boundary.

In this paper thick $(M \neq 0)$ and thin $(M=0)$ circular plate is considered and discussed its thermoelasticity with the help of the Goodier's thermoelastic displacement potential function and the Michell's function under steady temperature field. To obtain the temperature distribution integral transform method is applied. The results are obtained in series form in terms of Bessel's functions and the temperature change, displacement function and stresses have been computed numerically and illustrated graphically. Here we compute the effect of Michell function on the thickness of circular plate with internal heat generation. A mathematical model has been constructed for thick $(M \neq 0)$ and thin $(M=0)$ circular plate with the help of numerical illustration by considering aluminum (pure) circular plate. No one previously studied such type of problem. This is new contribution to the field.

The direct problem is very important in view of its relevance to various industrial mechanics subjected to heating such as the main shaft of lathe, turbines and the role of rolling mill, base of furnace of boiler of a thermal power plant, gas power plant.

\section{Formulation of the problem}

Consider a thick $(M \neq 0)$ circular plate of radius a and thickness h defined by $0 \leq r \leq a, \frac{-h}{2} \leq z \leq \frac{h}{2}$. The initial temperature in a thick circular plate is zero. The heat flux $-Q f(r)$ is applied on the upper surface of plate $\left(z=\frac{h}{2}\right)$ and the lower surface ( $z=-\frac{h}{2}$ ) is at temperature zero. The fixed circular edge $(r=a)$ is thermally insulated. Assume the circular boundary of a thick circular plate is free from traction. Under these prescribed conditions, the thermal steady state temperature, displacement and stresses in a thick circular plate with internal heat generation are required to be determined.

The differential equation governing the displacement potential function $\phi(r, z)$ is given by,

$$
\frac{\partial^{2} \phi}{\partial r^{2}}+\frac{1}{r} \frac{\partial \phi}{\partial r}+\frac{\partial^{2} \phi}{\partial z^{2}}=K \boldsymbol{\tau}
$$

where $\mathrm{K}$ is the restraint coefficient and temperature change $\tau=T-T_{i}, T_{i}$ is initial temperature. Displacement function $\phi$ is known as Goodier's thermoelastic displacement potential.

Temperature of the plate at time $t$ satisfying heat conduction equation as follows, 


$$
\frac{\partial^{2} T}{\partial r^{2}}+\frac{1}{r} \frac{\partial T}{\partial r}+\frac{\partial^{2} T}{\partial z^{2}}+\frac{q}{k}=0
$$

with the boundary conditions

$$
\begin{aligned}
& T=0 \text { at } \mathrm{z}=-\frac{h}{2}, \quad 0 \leq r \leq a \\
& \lambda \frac{\partial T}{\partial Z}=-\mathrm{Q} f(r) \text { at } z=\frac{h}{2}, 0 \leq r \leq a \\
& \frac{\partial T}{\partial r}=0 \text { at } r=a
\end{aligned}
$$

where $\alpha$ is the thermal diffusivity of the material of the plate, $\mathrm{k}$ is the thermal conductivity of the material of the plate and $\mathrm{q}$ is internal heat generation.

The Michell's function M must satisfy

$$
\nabla^{2} \nabla^{2} M=0
$$

where

$$
\nabla^{2}=\frac{\partial^{2}}{\partial r^{2}}+\frac{1}{r} \frac{\partial}{\partial r}+\frac{\partial^{2}}{\partial z^{2}}
$$

The components of the stresses are represented by the thermoelastic displacement potential $\phi$ and Michell's function $\mathrm{M}$ as

$$
\begin{aligned}
\sigma_{r r} & =2 G\left\{\frac{\partial^{2} \phi}{\partial r^{2}}-K \tau+\frac{\partial}{\partial z}\left[v \nabla^{2} M-\frac{\partial^{2} M}{\partial r^{2}}\right]\right\} \\
\sigma_{\theta \theta} & =2 G\left\{\frac{1}{r} \frac{\partial \phi}{\partial r}-K \tau+\frac{\partial}{\partial z}\left[v \nabla^{2} M-\frac{1}{r} \frac{\partial M}{\partial r}\right]\right\} \\
\sigma_{z z} & =2 G\left\{\frac{\partial^{2} \phi}{\partial z^{2}}-K \tau+\frac{\partial}{\partial z}\left[(2-v) \nabla^{2} M-\frac{\partial^{2} M}{\partial z^{2}}\right]\right\}
\end{aligned}
$$

and

$$
\sigma_{r z}=2 G\left\{\frac{\partial^{2} \phi}{\partial r \partial z}+\frac{\partial}{\partial r}\left[(1-v) \nabla^{2} M-\frac{\partial^{2} M}{\partial z^{2}}\right]\right\}
$$

where $\mathrm{G}$ and $\mathrm{v}$ are the shear modulus and Poisson's ratio respectively.

For traction free surface stress functions

$$
\sigma_{r r}=\sigma_{z z}=\sigma_{r z}=0 \text { at } z=-\frac{h}{2}
$$

\subsection{Temperature change}

\section{Solution}

To obtain the expression for temperature $T(r, z)$, we introduce the finite Hankel transform over the variable $\mathrm{r}$ and its inverse transform defined by [9] as

$$
\begin{array}{r}
\bar{T}\left(\beta_{m}, z\right)=\int_{r=0}^{a} r K_{0}\left(\beta_{m}, r\right) T(r, z) d r \\
T(r, z)=\sum_{m=1}^{\infty} K_{0}\left(\beta_{m}, r\right) \bar{T}\left(\beta_{m}, z\right)
\end{array}
$$

where $K_{0}\left(\beta_{m}, r\right)=\frac{R_{0}\left(\beta_{m}, r\right)}{\sqrt{N}}$,

$$
K_{0}\left(\beta_{m}, r\right)=\frac{\sqrt{2}}{a} \frac{J_{0}\left(\beta_{m} r\right)}{J_{0}\left(\beta_{m} a\right)}
$$

Eigen value $\beta_{m}$ are the positive root of $J_{0}{ }^{\prime}\left(\beta_{m} a\right)=0$

$\beta_{1}, \beta_{2} \ldots$. are roots of transcendental equation.

where $J_{n}(x)$ is Bessel function of the first kind of order $n$.

On applying the finite Hankel transform defined in the Eq. (13) and its inverse transform defined in (14) to the Eq. (2), one obtains the expression for temperature as

$$
\begin{aligned}
T(r, z)= & \sum_{m=1}^{\infty} \frac{\sqrt{2}}{a} \frac{J_{0}\left(\beta_{m} r\right)}{J_{0}\left(\beta_{m} a\right)}\left\{\frac{-A\left(\beta_{\mathrm{m}},-\frac{h}{2}\right) \cosh \left[\beta_{m}\left(z-\frac{h}{2}\right)\right]}{\cosh \left(\operatorname{hi} \beta_{m}\right)}\right. \\
& \left.+\left(\frac{-Q F\left(\beta_{m}\right)}{\beta_{m} \lambda}-\frac{1}{\beta_{m}} \frac{\partial A\left(\beta_{m}, \frac{h}{2}\right)}{\partial Z}\right) \frac{\sinh \left[\beta_{m}\left(z+\frac{h}{2}\right)\right]}{\cosh \left(\ln _{m}\right)}+A\left(\beta_{m}, z\right)\right\}
\end{aligned}
$$

$A\left(\beta_{m}, z\right)$ is particular integral of differential equation (2).

Michells function $\mathrm{M}$

Now suitable form of $M$ which satisfy Eq. (6) is given by

$$
M=\sum_{m=1}^{\infty} J_{0}\left(\beta_{m} r\right)\left\{B_{m} \cosh \left[\beta_{m}\left(z+\frac{h}{2}\right)\right]+C_{m} \beta_{m}\left(z+\frac{h}{2}\right) \sinh \left[\beta_{m}\left(z+\frac{h}{2}\right)\right]\right\}
$$

where $B_{m}$ and $C_{m}$ are arbitrary functions.

\subsection{Goodiers Thermoelastic Displacement Potential $\phi$}

Assuming the displacement function $\phi(r, z)$ which satisfies Eq. (1) as

$$
\phi(r, z)=\sum_{m=1}^{\infty} \frac{\sqrt{2}}{a} \frac{J_{0}\left(\beta_{m} r\right)}{J_{0}\left(\beta_{m} a\right)}\left\{\frac{-A\left(\beta_{\mathrm{m}},-\frac{h}{2}\right) \cosh \left[\beta_{m}\left(z-\frac{h}{2}\right)\right]}{\cosh \left(h \beta_{m}\right)}\right.
$$




$$
\left.+\left(\frac{-\mathrm{Q} F\left(\beta_{m}\right)}{\beta_{m} \lambda}-\frac{1}{\beta_{m}} \frac{\partial \mathrm{A}\left(\beta_{\mathrm{m}}, \frac{h}{2}\right)}{\partial Z}\right) \frac{\sinh \left[\beta_{m}\left(z+\frac{h}{2}\right)\right]}{\cosh \left(h \beta_{m}\right)}+A\left(\beta_{\mathrm{m}},-\frac{h}{2}\right) e^{\beta_{m}\left(z+\frac{h}{2}\right)}\right\}
$$

Now using Eqs. (18), (19) and (20) in Eq. (8), (9), (10) and (11), one obtains the expressions for stresses respectively as

$$
\begin{aligned}
& \frac{\sigma_{r r}}{K}=2 G \sum_{m=1}^{\infty}\left\{\frac{-\sqrt{2} \beta_{m}{ }^{2} J_{1}{ }^{\prime}\left(\beta_{m} r\right)}{a J_{0}\left(\beta_{m} a\right)}\right. \\
& \times\left[\begin{array}{c}
\frac{-A\left(\beta_{m},-\frac{h}{2}\right) \cosh \left[\beta_{m}\left(z-\frac{h}{2}\right)\right]}{\cosh \left(h \beta_{m}\right)}+\left(\frac{-Q F\left(\beta_{m}\right)}{\beta_{m} \lambda}-\frac{1}{\beta_{m}} \frac{\partial A\left(\beta_{m}, \frac{h}{2}\right)}{\partial Z}\right) \\
\times \frac{\sinh \left[\beta_{m}\left(z+\frac{h}{2}\right)\right]}{\cosh \left(\beta_{m} h\right)}+A\left(\beta_{m},-\frac{h}{2}\right) e^{\beta_{m}\left(z+\frac{h}{2}\right)}
\end{array}\right] \\
& -\frac{\sqrt{2} J_{0}\left(\beta_{m} r\right)}{a J_{0}\left(\beta_{m} a\right)} \times\left[\begin{array}{c}
\frac{-A\left(\beta_{m},-\frac{h}{2}\right) \cosh \left[\beta_{m}\left(z-\frac{h}{2}\right)\right]}{\cosh \left(h \beta_{m}\right)}+\left(\frac{-Q F\left(\beta_{m}\right)}{\beta_{m} \lambda}-\frac{1}{\beta_{m}} \frac{\partial A\left(\beta_{m}, \frac{h}{2}\right)}{\partial Z}\right) \\
\times \frac{\sinh \left[\beta_{m}\left(z+\frac{h}{2}\right)\right]}{\cosh \left(\beta_{m} h\right)}+A\left(\beta_{m}, z\right)
\end{array}\right] \\
& \left.+\beta_{m}{ }^{2}\left[\begin{array}{c}
2 v J_{0}\left(\beta_{m} r\right) C_{m}+\left(C_{m}+B_{m}\right) J_{1}^{\prime}\left(\beta_{m} r\right) \sinh \left[\beta_{m}\left(z+\frac{h}{2}\right)\right] \beta_{m} \\
+C_{m} J_{1}{ }^{\prime}\left(\beta_{m} r\right)\left(z+\frac{h}{2}\right) \cosh \left[\beta_{m}\left(z+\frac{h}{2}\right)\right]
\end{array}\right]\right\} \\
& \frac{\sigma_{\theta \theta}}{K}=2 G \sum_{m=1}^{\infty}\left\{\frac{-\sqrt{2} \beta_{m} J_{1}\left(\beta_{m} r\right)}{a r J_{0}\left(\beta_{m} a\right)}\right. \\
& \times\left[\begin{array}{c}
\frac{-A\left(\beta_{m},-\frac{h}{2}\right) \cosh \left[\beta_{m}\left(z-\frac{h}{2}\right)\right]}{\cosh \left(h \beta_{m}\right)}+\left(\frac{-Q F\left(\beta_{m}\right)}{\beta_{m} \lambda}-\frac{1}{\beta_{m}} \frac{\partial A\left(\beta_{m}, \frac{h}{2}\right)}{\partial Z}\right) \\
\times \frac{\sinh \left[\beta_{m}\left(z+\frac{h}{2}\right)\right]}{\cosh \left(\beta_{m} h\right)}+A\left(\beta_{m},-\frac{h}{2}\right) e^{\beta_{m}\left(z+\frac{h}{2}\right)}
\end{array}\right] \\
& -\frac{\sqrt{2} J_{0}\left(\beta_{m} r\right)}{a J_{0}\left(\beta_{m} a\right)} \times\left[\begin{array}{c}
\frac{-A\left(\beta_{m},-\frac{h}{2}\right) \cosh \left[\beta_{m}\left(z-\frac{h}{2}\right)\right]}{\cosh \left(h \beta_{m}\right)}+\left(\frac{-Q F\left(\beta_{m}\right)}{\beta_{m} \lambda}-\frac{1}{\beta_{m}} \frac{\partial A\left(\beta_{m}, \frac{h}{2}\right)}{\partial Z}\right) \\
\times \frac{\sinh \left[\beta_{m}\left(z+\frac{h}{2}\right)\right]}{\cosh \left(\beta_{m} h\right)}+A\left(\beta_{m}, z\right)
\end{array}\right] \\
& +\beta_{m}^{2} \sinh \left[\beta_{m}\left(z+\frac{h}{2}\right)\right]\left[2 v \beta_{m} J_{0}\left(\beta_{m} r\right) C_{m}+\frac{J_{1}\left(\beta_{m} r\right)}{r} B_{m}\right] \\
& \left.+C_{m} \beta_{m}{ }^{2} \frac{J_{1}\left(\beta_{m} r\right)}{r}\left[\sinh \left[\beta_{m}\left(z+\frac{h}{2}\right)\right]+\beta_{m}\left(z+\frac{h}{2}\right)^{r} \cosh \left[\beta_{m}\left(z+\frac{h}{2}\right)\right]\right]\right\} \\
& \frac{\sigma_{Z Z}}{K}=2 G \sum_{m=1}^{\infty}\left\{\frac{\sqrt{2} \beta_{m}{ }^{2} J_{0}\left(\beta_{m} r\right)}{a J_{0}\left(\beta_{m} a\right)}\right. \\
& \times\left[\begin{array}{c}
\frac{-A\left(\beta_{m},-\frac{h}{2}\right) \cosh \left[\beta_{m}\left(z-\frac{h}{2}\right)\right]}{\cosh \left(h \beta_{m}\right)}+\left(\frac{-Q F\left(\beta_{m}\right)}{\beta_{m} \lambda}-\frac{1}{\beta_{m}} \frac{\partial A\left(\beta_{m}, \frac{h}{2}\right)}{\partial Z}\right) \\
\times \frac{\sinh \left[\beta_{m}\left(z+\frac{h}{2}\right)\right]}{\cosh \left(\beta_{m} h\right)}+A\left(\beta_{m},-\frac{h}{2}\right) e^{\beta_{m}\left(z+\frac{h}{2}\right)}
\end{array}\right] \\
& -\frac{\sqrt{2} J_{0}\left(\beta_{m} r\right)}{a J_{0}\left(\beta_{m} a\right)} \times\left[\begin{array}{c}
\frac{-A\left(\beta_{m},-\frac{h}{2}\right) \cosh \left[\beta_{m}\left(z-\frac{h}{2}\right)\right]}{\cosh \left(h \beta_{m}\right)}+\left(\frac{-Q F\left(\beta_{m}\right)}{\beta_{m} \lambda}-\frac{1}{\beta_{m}} \frac{\partial A\left(\beta_{m}, \frac{h}{2}\right)}{\partial Z}\right) \\
\times \frac{\sinh \left[\beta_{m}\left(z+\frac{h}{2}\right)\right]}{\cosh \left(\beta_{m} h\right)}+A\left(\beta_{m}, z\right)
\end{array}\right] \\
& +\beta_{m}{ }^{3} \sinh \left[\beta_{m}\left(z+\frac{h}{2}\right)\right]\left[(1+2 v) C_{m}+B_{m}\right] \\
& \left.-C_{m} \beta_{m}{ }^{4}\left(z+\frac{h}{2}\right) \cosh \left[\beta_{m}\left(z+\frac{h}{2}\right)\right]\right\} \\
& \frac{\sigma_{r z}}{K}=2 G \sum_{m=1}^{\infty} \beta_{m}^{2} J_{1}\left(\beta_{m} r\right)\left\{\frac{-\sqrt{2}}{a J_{0}\left(\beta_{m} a\right)}\right. \\
& \times\left[\begin{array}{c}
\frac{-A\left(\beta_{m},-\frac{h}{2}\right) \sinh \left[\beta_{m}\left(z-\frac{h}{2}\right)\right]}{\cosh \left(h \beta_{m}\right)}+\left(\frac{-Q F\left(\beta_{m}\right)}{\beta_{m} \lambda}-\frac{1}{\beta_{m}} \frac{\partial A\left(\beta_{m}, \frac{h}{2}\right)}{\partial Z}\right) \\
\times \frac{\cosh \left[\beta_{m}\left(z+\frac{h}{2}\right)\right]}{\cosh \left(\beta_{m} h\right)}+A\left(\beta_{m},-\frac{h}{2}\right) e^{\beta_{m}\left(z+\frac{h}{2}\right)}
\end{array}\right] \\
& \left.+\left(2 v C_{m}+B_{m}\right) \beta_{m} \cosh \left[\beta_{m}\left(z+\frac{h}{2}\right)\right]-C_{m} \beta_{m}^{2}\left(z+\frac{h}{2}\right) \sinh \left[\beta_{m}\left(z+\frac{h}{2}\right)\right]\right\}
\end{aligned}
$$

In order to satisfy condition (12), solving equations (21), (23) and (25) for $B_{m}$ and $C_{m}$ one obtains

Let $C_{m}=0$ 


$$
B_{m}=\frac{\sqrt{2}}{a \beta_{m} J_{0}\left(\beta_{m} a\right)}\left[\begin{array}{c}
\frac{A\left(\beta_{m},-\frac{h}{2}\right) \sin h\left(\beta_{m} h\right)}{\cos h\left(\beta_{m} h\right)}+\left(\frac{-Q F\left(\beta_{m}\right)}{\beta_{m} \lambda}-\frac{1}{\beta_{m}} \frac{\partial A\left(\beta_{m}, \frac{h}{2}\right)}{\partial Z}\right) \\
\times \frac{1}{\cos h\left(\beta_{m} h\right)}+A\left(\beta_{m},-\frac{h}{2}\right)
\end{array}\right]
$$

Setting

\section{Special case and Numerical calculations}

(1) $\mathrm{f}(\mathrm{r})=\delta\left(r-r_{0}\right)$

$$
a=1 m, h=0.25 m, r_{0}=1 m
$$

where $\delta(r)$ is well known diract delta function of argument $\mathrm{r}$.

$$
\begin{aligned}
& F\left(\beta_{m}\right)=\frac{\sqrt{2}}{a} r_{0} J_{0}\left(\beta_{m} r_{0}\right) \\
& q=\delta(r-0.5) z \\
& \bar{q}=\int_{r=0}^{a} r \frac{\sqrt{2}}{a} \frac{J_{0}\left(\beta_{m} r\right)}{J_{0}\left(\beta_{m} a\right)} \delta(r-0.5) z d r \\
& =\frac{\sqrt{2}}{a} \frac{J_{0}\left(\beta_{m} 0.5\right)(0.5)}{J_{0}\left(\beta_{m} a\right)} z
\end{aligned}
$$

For thick plate $h=0.25 \mathrm{~m}$ and for thin plate $h=0.2 \mathrm{~m}$.

\section{Material Properties}

The numerical calculation has been carried out for aluminum (pure) circular plate with the material properties defined as

Thermal diffusivity $\alpha=84.18 \times 10^{-6} \mathrm{~m}^{2} \mathrm{~s}^{-1}$,

Specific heat $c_{\rho}=896 \mathrm{~J} / \mathrm{kg}$,

Thermal conductivity $\mathrm{k}=204.2 \mathrm{~W} / \mathrm{mK}$,

Shear modulus $G=25.5 G p a$,

Poisson ratio $\vartheta=0.35$.

\section{Roots of Transcendental Equation}

The $\beta_{1}=3.8317, \beta_{2}=7.0156, \beta_{3}=10.1735, \beta_{4}=13.3237, \beta_{5}=16.4706, \beta_{6}=19.6159$ are the roots of transcendental equation $J_{0}{ }^{\prime}\left(\beta_{m} a\right)=0$. The numerical calculation and the graph has been carried out with the help of mathematical software Matlab.

\section{Discussion}

In this paper a thick $(M \neq 0)$ and thin $(M=0)$ circular plate is considered and determined the expressions for stresses due to internal heat generation within it and we compute the effect of Michell function on the thickness of circular plate with internal heat generation along the radial direction. As a special case mathematical model is constructed for considering aluminum (pure) circular plate with the material properties specified above.

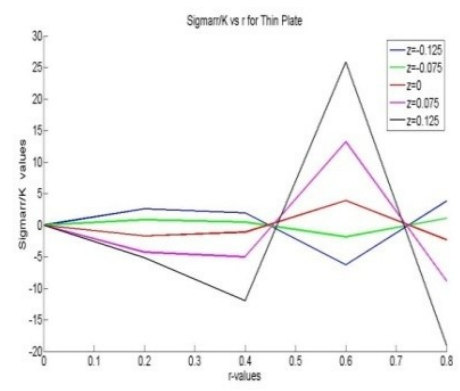

Fig. 1 Radial stress function $\frac{\sigma_{\mathrm{rr}}}{\mathrm{K}}$ for $(M=0)$.

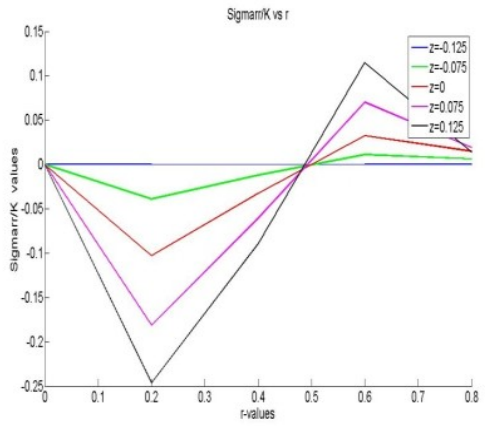

Fig. 2 Radial stress function $\frac{\sigma_{\mathrm{rr}}}{\mathrm{K}}$ for $(M \neq 0)$. 


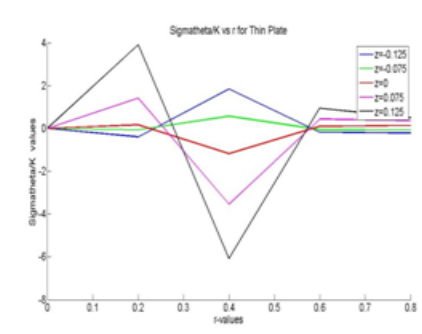

Fig. 3 Angular stress function $\frac{\sigma_{\theta 0}}{\mathrm{~K}}$ for $(M=0)$.

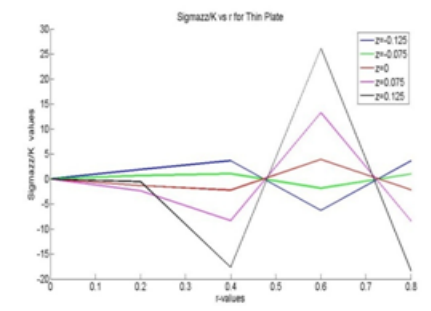

Fig. 5 Axial stress function $\frac{\sigma_{z z}}{\mathrm{~K}}$ for $(M=0)$.

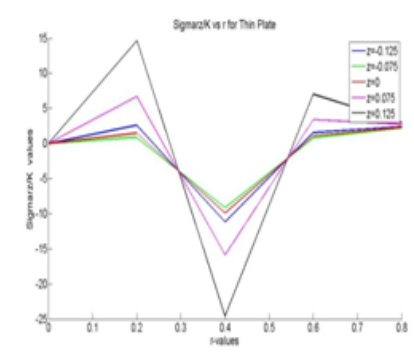

Fig. 7 Stress function $\frac{\sigma_{\mathrm{rz}}}{\mathrm{K}}$ for $(M=0)$.

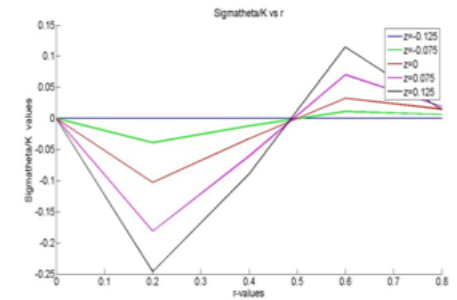

Fig. 4 Angular stress function $\frac{\sigma_{\text {鵤 }}}{\mathrm{K}}$ for $(M \neq 0)$.

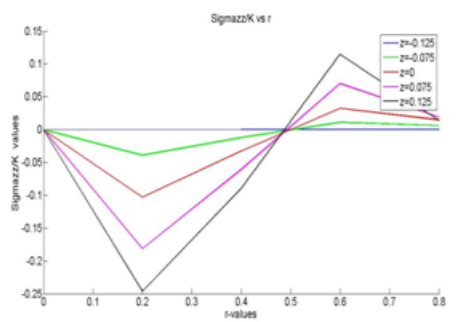

Fig. 6. Axial stress function $\frac{\sigma_{z z}}{\mathrm{~K}}$ for $(M \neq 0)$.

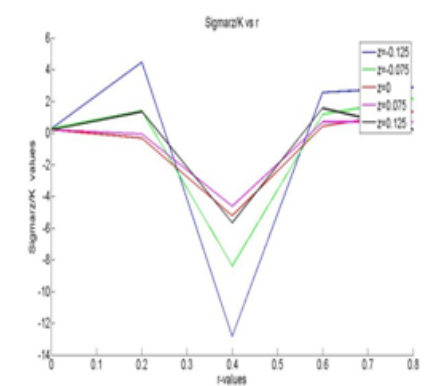

Fig. 8 Stress function $\frac{\sigma_{\mathrm{rz}}}{\mathrm{K}}$ for $(M \neq 0)$.

From Fig. 1 and 2, it is observed that due to Michell function the radial stress function $\sigma_{r r}$ increases towards the lateral surface of thick circular plate along radial direction, it develops tensile stress in the radial direction.

From Fig. 3 and 4, it is observed that due to Michell function the angular stress function $\sigma_{\theta \theta}$ increases towards the lateral surface of thick circular plate along radial direction, it develops tensile stress in the radial direction.

From Fig. 5 and 6, it is observed that due to Michell function the axial stress function $\sigma_{z z}$ increases towards the lateral surface of thick circular plate along radial direction, it develops tensile stress in the radial direction.

From Fig. 7 and 8, it is observed that due to Michell function the stress function $\sigma_{r z}$ decreases towards the lateral surface of thick circular plate along radial direction, it develops compressive stress in the radial direction.

\section{Conclusion}

We can summarize that in case of steady state behavior the radial stress function $\sigma_{r r}$, angular stress function $\sigma_{\theta \theta}$ and axial stress function $\sigma_{z z}$ increases towards the lateral surface of thick circular plate along radial direction. Also the stress function $\sigma_{r z}$ decreases towards the lateral surface of thick circular plate along radial direction. The Michell function act as a moderant with the thickness of circular plate.

The results obtained here are useful in engineering problems particularly in the determination of state of stress in thick circular plate and base of furnace of boiler of a thermal power plant and gas power plant.

\section{References}

[1] W. Nowacki, The state of stresses in a thick circular disk due to temperature field, Bull. Acad. Polon. Sci., Ser. Sci. Techn., 5, 227, (1957).

[2] Roy Choudhary S. K., A note of quasi static stress in a thin circular plate due to transient temperature applied along the circumference of a circle over the upper face, Bull Acad. Polon Sci, Ser, Scl, Tech., 20-21, (1972).

[3] J. N. Sharma, P. K. Sharma and R. L. Sharma, Behavior of thermoelastic thick plate under lateral loads, Journal of Thermal Stresses, 27, 171-191, (2004). 
[4] V. S. Gogulwar and K. C. Deshmukh, Thermal stresses in a thin circular plate with heat sources, Journal of Indian Academy of Mathematics, 27, (1), (2005).

[5] V. S. Kulkarni and K. C. Deshmukh, Quasi-static thermal stresses in steady state thick circular plate, Journal of Brazilian Society of Mechanical Sciences and Engineering, XXX, (2) 175, (2008).

[6] K.C. Deshmukh, S. D. Warbhe and V.S. Kulkarni, Non-homogeneous steady state heat conduction problem in a thin circular plate and thermal stresses, Int. J. Thermophysics, 30, 1688-1696, (2009).

[7] K. C. Deshmukh, Y. I. Quazi, S. D. Warbhe and V. S Kulkarni, Thermal stresses induced by a point heat source in acircular plate by quasi static approach, Theoretical and Applied Mechanics Letters 1, 031007, (2011).

[8] Naotake Noda, Richard B Hetnarski and Yoshinobu Tanigawa, Thermal stresses 2 nd edn., 259-261, Taylor and Francis New York, (2003).

[9] M. N. Ozisik, Boundary value problems of heat conduction International Text Book Company, Scranton, Pennsylvania, (1968). 